\title{
Electric-field-induced submicrosecond resistive switching
}

\author{
N. Das, ${ }^{1}$ S. Tsui, ${ }^{1}$ Y. Y. Xue, ${ }^{1}$ Y. Q. Wang, ${ }^{1}$ and C. W. $\mathrm{Chu}^{1,2,3}$ \\ ${ }^{1}$ Department of Physics and TCSUH, University of Houston, Houston, Texas 77204-5002, USA \\ ${ }^{2}$ Hong Kong University of Science and Technology, Hong Kong \\ ${ }^{3}$ Lawrence Berkeley National Laboratory, 1 Cyclotron Road, Berkeley, California 94720, USA
}

(Received 23 July 2008; revised manuscript received 30 September 2008; published 10 December 2008)

\begin{abstract}
Electric-field-induced resistive switching in metal-oxide interfaces has attracted extensive recent interest. While many agree that lattice defects play a key role, details of the physical processes are far from clear. There is debate, for example, regarding whether the electromigration of pre-existing point defects or the field-created larger lattice defects dominates the switch. We investigate several Ag- $\mathrm{Pr}_{0.7} \mathrm{Ca}_{0.3} \mathrm{MnO}_{3}$ samples exhibiting either submicrosecond fast switching or slow quasistatic de switching. It is found that the carrier trapping potentials are very different for the pre-existing point defects associated with doping (and/or electromigration) and for the defects responsible for the submicrosecond fast switching. Creation/removal of the defects with more severe lattice distortions and spatial spreading (trapping potential $\geq 0.35 \mathrm{eV}$ ), therefore, should be the dominating mechanism during submicrosecond switching. On the other hand, the shallow defects (trapping potential $\ll 0.2 \mathrm{eV}$ ) associated with doping/annealing are most likely responsible for the resistance hysteresis (slow switch) during quasistatic voltage sweep.
\end{abstract}

DOI: 10.1103/PhysRevB.78.235418

PACS number(s): 73.20.Hb, 68.55.Ln, 77.22.Gm

\section{INTRODUCTION AND MOTIVATION}

Electric-field-induced resistive switching between nonvolatile high resistance state (HRS) and low resistance state (LRS) across metal-oxide interfaces has been studied extensively for the potential application in memory devices for resistive random access memory (RRAM). Different oxides, ranging from perovskites ${ }^{1,2}$ to binary oxide materials, have been found to show similar effects. ${ }^{3}$ Many different models, including metal-insulator transition, ${ }^{4}$ electromigration, ${ }^{5,6}$ and lattice rearrangement, ${ }^{7}$ have been suggested to explain the physical mechanism behind the switch. However, developing a self-consistent model to accommodate key observations has proven to be difficult. Many groups, for example, attribute the submicrosecond fast switching observed in various metal-oxide interfaces, e.g., $\quad \mathrm{Ag}_{-} \mathrm{Pr}_{0.7} \mathrm{Ca}_{0.3} \mathrm{MnO}_{3}$ (PCMO), to the same electromigration mechanism, i.e., changes in the doping level, proposed for the reversible slow resistance change in bulk $\mathrm{SrTiO}_{3}$ (STO). ${ }^{6}$ Unusually large diffusion coefficients were proposed to support the idea. Unfortunately, such interpretations may contradict the well established Nernst-Einstein (NE) relation even if the parameters can be arbitrarily chosen. Both the thermal ion diffusion, which determines the retention (stability) of the alternated resistance state, and the electromigration, which is suggested to cause the switching, are based on the same physical process in the NE relation. The retention time $t_{R}$ and the switching speed $\tau$, therefore, are intrinsically related. The electromigration flux, $J_{E}$, and the thermal diffusion flux, $J_{D}$, across a layer of thickness $\Delta x$ are $D n Z e E /(k T)$ and $D \cdot d n / d x \approx D \cdot \Delta n / \Delta x$, respectively, where $D, n, e, E, \Delta n$, and $Z$ are the diffusion constant, the defect density, the electron charge, the local electric field, the $n$ difference across the interface layer, and the effective charge number, respectively. Therefore, the ratio $t_{R} / \tau=\frac{J_{E}}{J_{D}}=\frac{V_{F} Z e}{k T} \cdot \frac{n}{\Delta n} \ll 10^{9}$, which is independent of both $D$ and $\Delta x$ based on the exaggerated values of the applied voltage $V_{F}=E \cdot \Delta x=100 \mathrm{~V}, Z=10, n$
$=1 /$ cell, and $\Delta n=0.01 /$ cell. If the electromigration of preexisting point defects is responsible for fast submicrosecond switching, it can hardly give a self-consistent description from the viewpoint of NE relation. Based on reported fast switches possessing $t_{R}>10^{8} \mathrm{sec}$ (a very good retention) and $\tau=10^{-7}$ (a very fast switching speed), the ratio $\frac{J_{E}}{J_{D}} \approx 10^{15}$ far exceeds the theoretical limit set by the NE relation.

The discrepancy related to the NE relation mentioned above motivated our current study of the mechanism related to fast switching. The transport property across the interface and its variation with the switching should be a natural starting point to explore the underlying mechanisms. In particular, the carrier trapping potentials at the interface should be a fingerprint of the defects involved. The situation, however, is complicated by the diversity in the reported transport characteristics. In the case of Ag-PCMO, for example, three broad classes have been reported: (1) Schottky-like ${ }^{8}$ rectifying $I-V,(2)$ carrier hopping $(\mathrm{CH})$ (Ref. 9), and (3) spacecharge limited conduction (SCLC). ${ }^{10-12}$ In the following study, we will concentrate on the SCLC-type sample conduction in PCMO and try to shed light on the nature of the defects responsible for the fast submicrosecond switch, which appear to be decisively different from the defects associated with the quasistatic hysteresis.

\section{EXPERIMENTAL DETAILS}

The samples used here are PCMO films on $\mathrm{LaAlO}_{3}$ substrates with $\mathrm{Ag}$ electrodes (width $=0.5 \mathrm{~mm}$, length $=4 \mathrm{~mm}$ ) deposited ex situ. The PCMO films (thickness $\approx 400 \mathrm{~nm}$ ) deposited through ac sputtering are epitaxial with the preferred orientation based on the X-ray diffraction (XRD) data. A parallel three-electrode configuration (Fig. 1), which removes the contribution from the bulk regions of the sample, is used to measure the interface resistance $R$ and capacitance $C{ }^{13}$ The switching was induced through a $200 \mathrm{~ns}$ pulse (pulse voltage up to $100 \mathrm{~V}$ ) generated by a DEI PVX-4150 


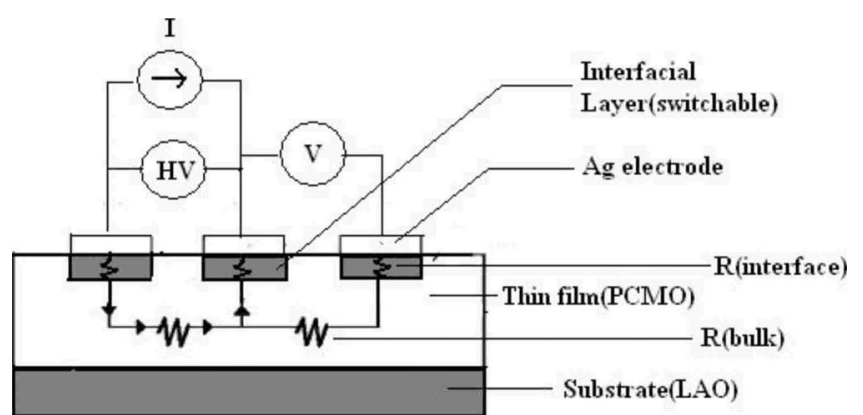

FIG. 1. A schematic of the three-lead configurations used to measure the interfacial resistance and capacitance in the Ag-PCMO interface. HV pulses are first applied to induce a switch. Immediately after, a small measurement current $(i)$ is applied while the voltage drop $(v)$ is measured between the second lead and a reference electrode, thereby measuring the interfacial resistance.

function generator acting as a gate for a Keithley 2400 source meter. The actual pulse voltage drop across the interface layer, which is different from applied pulse voltage ( $\sim 50 \mathrm{~V}$ for fast switching samples), was measured using an HP54502A digital oscilloscope through the same three-lead configuration. Immediately after pulsing, a small measurement current $I(1 \mu \mathrm{A})$ was used to measure the interfacial $R$ with a HP34401A multimeter. The impedances were measured using an SR-830 lock-in amplifier (swept from $1-10^{5} \mathrm{~Hz}$ ) and a Solartron 1260 impedance analyzer (up to $10^{7} \mathrm{~Hz}$ ), again using the three-lead configuration with an excitation voltage of $100 \mathrm{mV}$.

\section{RESULTS AND DISCUSSION}

In Fig. 2, the logarithmic $\left(\log _{10} I-\log _{10} V\right)$ plot of the three-lead $I-V$ for both LRS and HRS can be roughly divided into two distinct regions: Ohmic (up to $0.1 \mathrm{~V}$ ) and a distinct SCLC region with $I \propto V^{n}$ at higher voltages. The index $n$ is

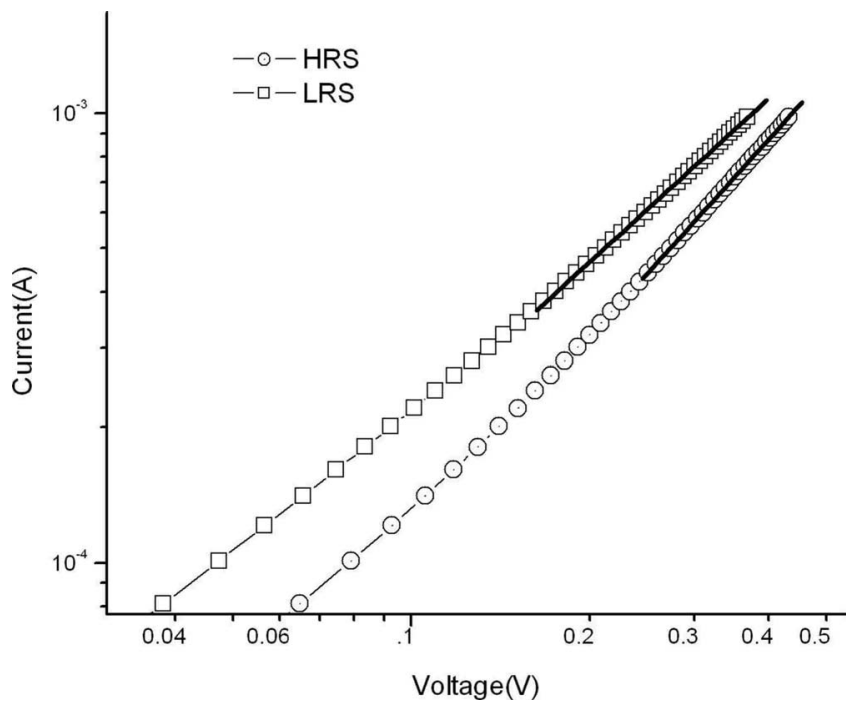

FIG. 2. $I-V\left(\log _{10} I-\log _{10} V\right)$ for PCMO-Ag interface showing SCLC. Open circles denote the HRS, whereas open squares denote the LRS. The lines above $0.2 \mathrm{~V}$ are the power-law fits. $\approx 1.4$ at $0.3 \mathrm{~V}$ for HRS in Fig. 2, and increases with the applied voltage (not shown). In a few cases, where the higher resistance permits the measurement to be extended to $2 \mathrm{~V}$, the $n$ can even reach two. This is typical behavior of thin disordered layers below the so-called trap-filling limit, i.e., with a significant fraction of the traps still unfilled. Following the simplified SCLC model of Rose ${ }^{14}$ assuming an accept/donor concentration $N_{a}$, we can write the current $I$ at low frequencies as

$$
I=N_{a} e \mu_{0} V 2 \theta / d
$$

at the Ohmic region, where $\theta=\left(N_{c} / 2 N_{t}\right) e^{-U / K T}, \mu_{0}, N_{c}, N_{a}$, $U, N_{t}, k T, V, d$, and $e$ are the fraction of trapped carriers, the carrier mobility, the density of states in the valence band, the acceptor density, the effective trapping potential, the number of traps, Boltzmann's constant, the temperature, the applied voltage, the effective thickness, and the electronic charge, respectively. Similarly, the quasistatic current at the SCLC region is

$$
I=\left(\varepsilon \mu_{0} \theta / d^{3}\right) V^{2},
$$

where $\varepsilon$ is dielectric constant. Assuming drift mobility $\mu_{0}$ does not change during switching, the resistance change between the HRS and the LRS will be naturally interpreted as the change of $\theta$, as previously suggested. ${ }^{7}$ The absence of the sudden $R$ jump with $V$ (sudden change in the slope of the $I-V$ curve) demonstrates that the trap-filled limit has not been reached here. ${ }^{11}$

As demonstrated by Eq. (2), the change in density of the deep defects between HRS and LRS can be extracted from our data, as previously done in Ref. 7. A rough estimation yields a 10-100 times change in defect density, which is further verified by capacitance measurement. The combined extraction of defect density change and the measured increased interfacial capacitance while changing from LRS to HRS $\left(C_{\mathrm{HRS}}>C_{\mathrm{LRS}}\right)$ suggests that a defect creation/ annihilation process dominates the fast submicrosecond switching in these samples.

It should be pointed out that two characteristics should appear under such a trap-dominating SCLC mechanism at a finite frequency $\omega$. First, an additional capacitance $C$ $=\operatorname{Im}[\mathbf{I}(\omega) / \mathbf{V}(\omega)] / \omega$ associated with the trapped carriers should appear in the SCLC region. Only geometric capacitance contributes in the Ohmic region without the unbalanced hot carriers. This capacitance component, i.e., all accumulated hot carriers, should be $\propto 1 / \theta$ at a given injected current, i.e., higher at HRS, as previously mentioned. Second, strong dispersions are expected for the complex $1 / \mathbf{Z}(\omega)=\mathbf{I}(\omega) / \mathbf{V}(\omega)=1 / R(\omega)-\boldsymbol{i} \omega C$, where $\mathbf{Z}, \mathbf{I}$, and $\mathbf{V}$ are the impedance, the applied current, and the voltage drop, respectively. Small-signal ac-response models have been proposed. ${ }^{15-17}$ While the trapped and free carriers should reach a thermal equilibrium at $\omega \ll \omega_{0}\left(\omega_{0}=v_{0} e^{-U / k T}\right)$, the carriers injected under an ac field with $\omega \gg \omega_{0}$ are unable to reestablish the space-charge distribution, which is needed to limit further current increase, where $v_{0}$ and $U$ are the socalled trial frequency on the order of $10^{11} \mathrm{~Hz}$ and the trapping potential, respectively. As a result, both $R(\omega)$ and $C(\omega)$ at higher $\omega$ will be lower than their respective low- $\omega$ values, 

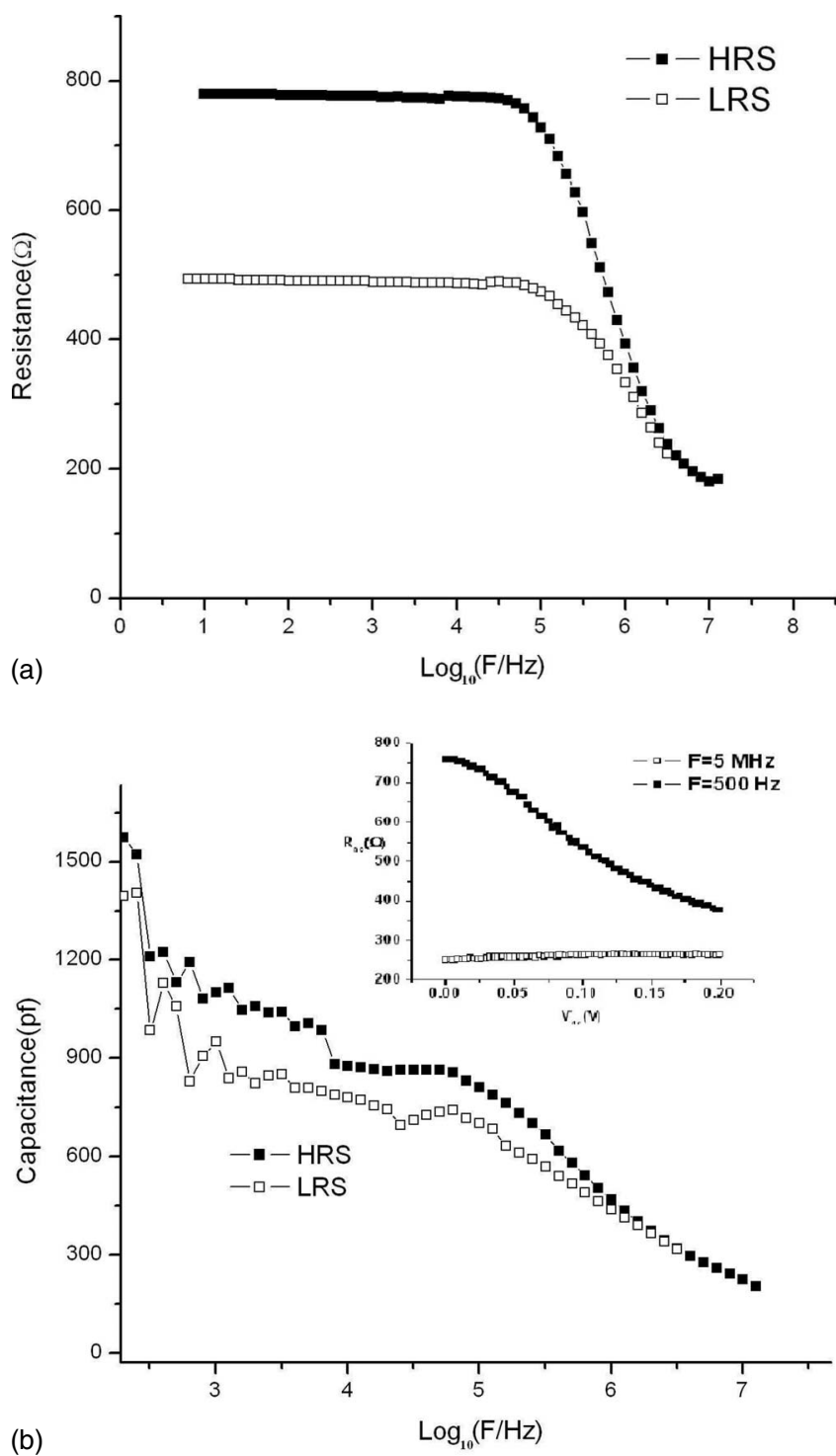

FIG. 3. Frequency dependence ( $\log _{10} F$ in a linear scale) of interfacial (a) resistance $[R(\omega)]$ and (b) capacitance $[C(\omega)]$ at zero bias for HRS (closed squares) and LRS (open squares). Inset: variation in $R_{\mathrm{ac}}$ under dc bias $\left(V_{\mathrm{dc}}\right)$ for HRS in PCMO-Ag interface. Closed squares $(f=500 \mathrm{~Hz})$ and open squares $(f=5 \mathrm{MHz})$ are below and above the threshold frequency $\left(\omega_{\mathrm{th}}=1 \mathrm{MHz}\right)$, respectively.

as will be shown later in our impedance spectra. In the Ohmic region, the situation is more complicated. However, similar dispersion is again found if the mean-free path is so short that the transport can be modeled as hopping among the defects. ${ }^{18}$ This offers a way to characterize the trapping potentials of the defects associated with the switching.

We therefore systematically explored the impedance spectroscopy of electrode-sample interfaces before and after switching, again adopting the three-lead configuration. ${ }^{13}$ In all our plots for frequency dependence, we have plotted $\log _{10} F$ in a linear scale in horizontal axis. A typical data set plotting the frequency dependence of the interfacial resistance $R(\omega)$ and capacitance $C(\omega)$ is shown in Fig. 3. Strong dispersions appear around $100 \mathrm{kHz}$ in both $R$ and $C$ data. The small split of the $C$ between HRS and LRS [Fig. 3(b)]

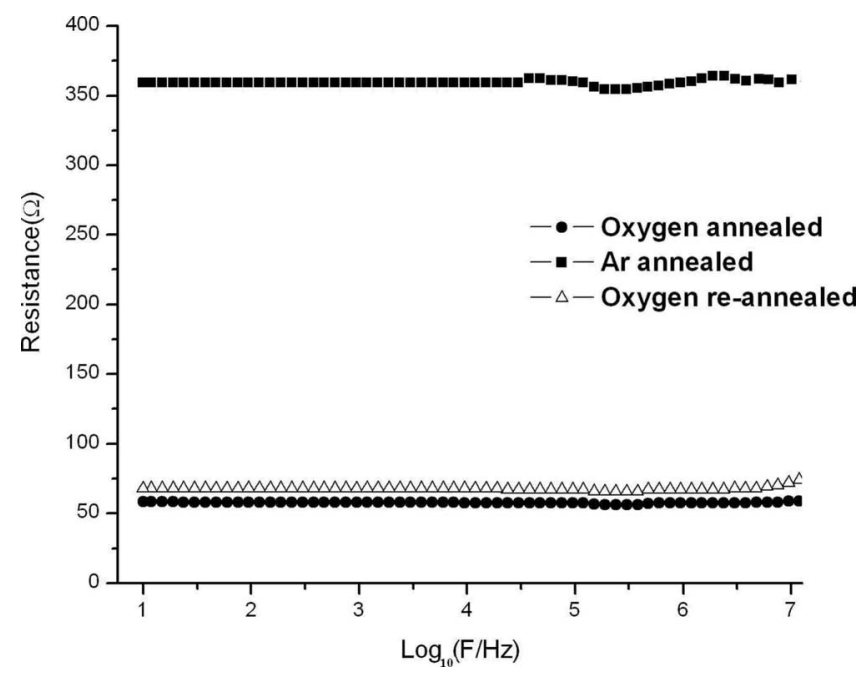

FIG. 4. Reversible change and frequency dependence $\left(\log _{10} F\right.$ in a linear scale) of interfacial resistance due to annealing. Closed circles denote an $\mathrm{O}_{2}$ annealed sample, closed squares denote $\mathrm{Ar}$ annealed sample, and open triangles denote $\mathrm{O}_{2}$ reannealed sample.

shows a noticeable contribution of the injected hot carriers. It is especially interesting to note that the resistance switching, i.e., the $R$ difference between HRS and LRS, [Fig. 3(a)] appears only at lower $\omega$. This shows that the switch is closely associated with the defects with trapping potential $U$ on the order of $k T \cdot \ln \left(v_{0} / \omega_{0}\right)$. In all samples tested so far (more than 20 , all switched by submicrosecond pulses), the apparent $\omega_{0}$ spreads from $100 \mathrm{kHz}$ to $1 \mathrm{MHz}$. The corresponding trapping potential, therefore, lies between $0.3-0.35 \mathrm{eV}$, i.e., relatively deep trapping centers.

To further explore the role of the threshold frequency $\omega_{0}$, we studied the field dependence of $R_{\mathrm{ac}}$ at $500 \mathrm{~Hz}$ and $5 \mathrm{MHz}$, which are far below and above the $\omega_{0}=1 \mathrm{MHz}$, respectively [inset of Fig. 3(b)]. The $500 \mathrm{~Hz} R_{\mathrm{ac}}$ are in good agreement with the previously obtained SCLC dc $I-V$ characteristic (Fig. 2). The power-law $E$ dependence clearly prefers a SCLC mechanism. Both the capacitance and its split between HRS and LRS increase with $E$, as expected from the SCLC models. The $E$ dependency at $5 \mathrm{MHz}$, however, is very different. The $R_{\mathrm{ac}}$ here not only breaks the power law but even increases with the bias $V_{\mathrm{dc}}$. This is in agreement with the model prediction that the injected carriers (which causes SCLC) can no longer reestablish the equilibrium space charge at the interface, at $\omega \gg v_{0} e^{-U / k T}$. This strongly supports our interpretation that the observed dispersion is associated with the carrier relaxation in the traps.

For comparison with the pre-existing point defects, primarily the oxygen and manganese vacancies as well as the interstitials in PCMO,${ }^{10}$ we carried out annealing experiments. Under proper annealing conditions, we can reversibly change the density of such point defects in the interface as well as in the bulk. An Ag-PCMO sample was first annealed in pure $\mathrm{O}_{2}$ at $500{ }^{\circ} \mathrm{C}$ for $6 \mathrm{~h}$ (oxidation) and subsequent reduction was performed in pure $\mathrm{Ar}$ gas at $500{ }^{\circ} \mathrm{C}$ for $6 \mathrm{~h}$, followed by another $\mathrm{O}_{2}$ annealing. The interface impedance was measured after each step, and the $R(\omega)$ extracted (Fig. 4). In addition to the expected reversible changes of the resistance, 
the impedance spectrum is clearly dispersionless up to 10 $\mathrm{MHz}$, which is the experimental upper limit, under both oxidized and reduced conditions. This is in clear contrast to the strong dispersion observed in Fig. 3. It is interesting to note the high interface resistances and the significant changes through the annealing, which should suggest both an extremely high density of the point defects and a significant doping effect of the annealing. We have also observed similar dispersionless features in bulk PCMO. It should be pointed out that these are in agreement with previously reported similarly weak $\omega$ dependency up to terahertz of the manganites at room temperature. ${ }^{19,20}$ The associated carrier trapping potentials, therefore, can be calculated in the same way as before, giving $V_{\text {trap }} \ll 0.2 \mathrm{eV}$ for the pre-existing point defects, in agreement with existing literature. ${ }^{19,20}$ Hence, the submicrosecond switching requires defects with much deeper trapping potentials through either more severe local lattice distortions and/or larger spatial spreading.

Motivated by the results of the annealing experiments, it is worthwhile to explore the Ag-PCMO quasistatic switch and the impedance spectrum during quasistatic sweep. A voltage pulse of $2 \mathrm{~s}$ duration was applied across the interface which was then followed by a resistance measurement, as before [Fig. 5(a)]. A subsequent pulse-then-measure series was performed, with the voltage pulses ramped between +15 and $-15 \mathrm{~V}$. It should be noted that these samples also exhibited SCLC behavior during a typical $I-V$ sweep (not shown) but there is no direct way to show that SCLC from this $R$ hysteresis plot [Fig. 5(a)] which is not an $I-V$ sweep. A hysteresis in the resistance does appear as the voltage passes over a threshold value. This is similar to the case of STO, ${ }^{6}$ where electromigration of pre-existing oxygen vacancies is proposed as the cause of the observed $R$ hysteresis. No fast switching has been observed in these dispersionless samples up to a voltage pulse of $300 \mathrm{~V}$ (compared to the $50 \mathrm{~V}$ submicrosecond pulses required to switch samples with strong dispersion). We suggest that this different behavior arises from the prevalent types of defects present. It should also be pointed out that no significant dispersion of $R$ was observed up to $10 \mathrm{MHz}$ at both HRS and LRS during the quasistatic voltage sweep [as a result of the shallow defects, Fig. 5(b)] but strong dispersions appear as soon as high-voltage (HV) pulses invoke submicrosecond switching, i.e., created deeper defects.

A slow switch also solves the apparent puzzle regarding the NE relation mentioned earlier. As the quasistatic switching time is on the order of seconds (or even minutes), the expected ratio $t_{R} / \tau$ is consistent with the NE formulism. The higher pulse voltage applied for $\mu s$ switching is so high that the carrier impact energy is far larger than $k T$ (at room temperature) but closer to the lattice bond energy. The NE (Nernst-Einstein) correlation, therefore, may no longer be applicable. While our frequency region prohibits us from verifying whether the defects associated with quasistatic switches are the same as the point defects responsible for carrier doping in PCMO, we were able to show clearly that the defects responsible for the submicrosecond switching are not such pre-existing point defects.

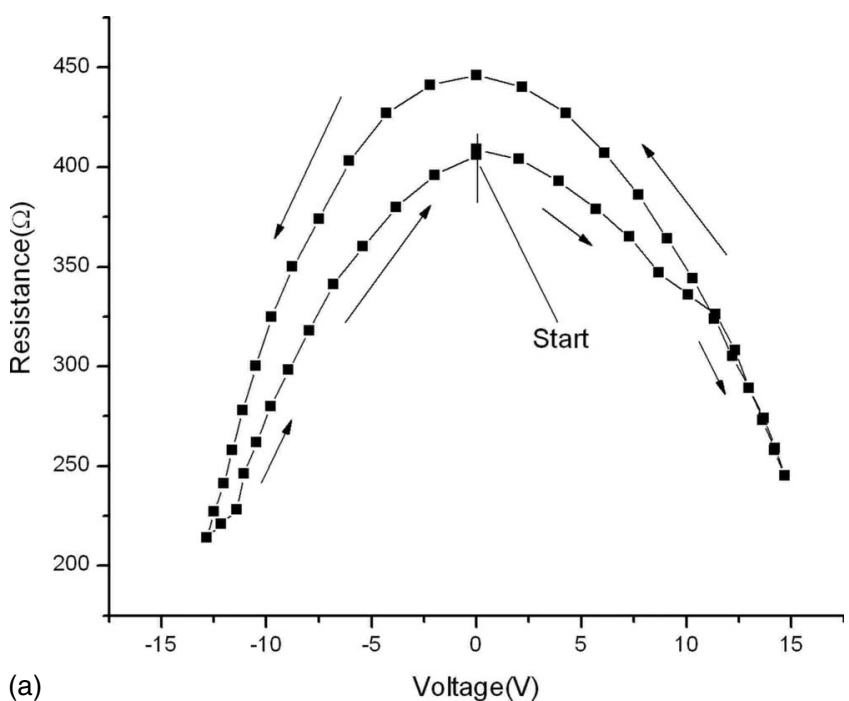

(a)

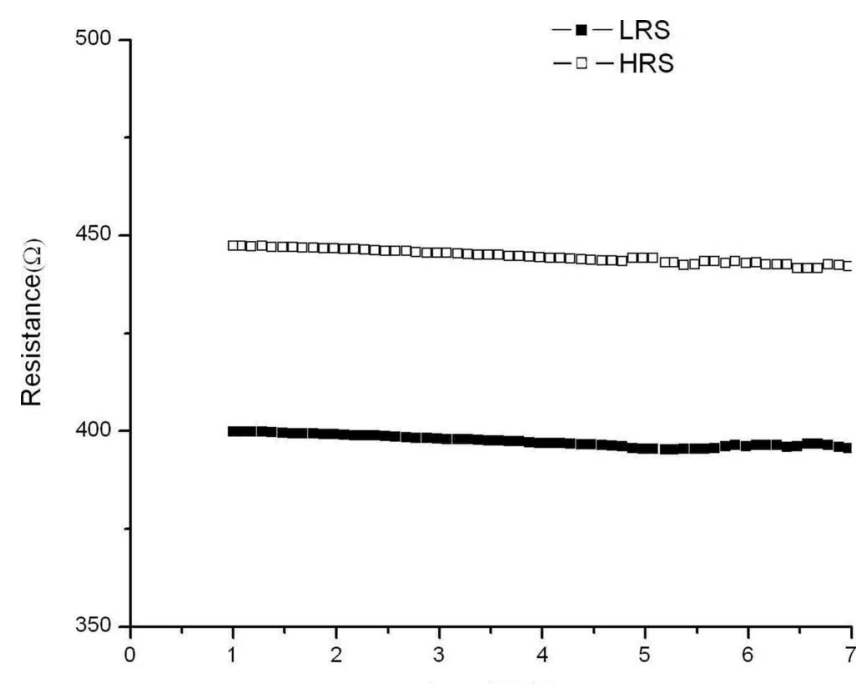

(b)

$\log _{10}(\mathrm{~F} / \mathrm{Hz})$

FIG. 5. (a) dc quasistatic slow switch in PCMO-Ag interface The data are obtained by applying a $2 \mathrm{~s}$ voltage pulse followed by a resistance measurement and then repeating the process as voltage is ramped up. It should be noted that this is not an $I-V$ curve. (b) Frequency dependence $\left(\log _{10} F\right.$ in a linear scale) of interfacial (a) resistance $[R(\omega)]$ and (b) capacitance $[C(\omega)]$ of the high (open squares) and low (closed squares) resistance states established from the quasistatic sweep.

\section{CONCLUSION}

In conclusion, we have shown that a change of doping level (density of oxygen vacancies or cationic defects) cannot explain the fast submicrosecond switching present in the metal electrode-PCMO system. Field-induced fast switching in PCMO has a definite nonshallow defect origin. These deep defects responsible for switching have a very high potential (trap) barrier $\geq 0.35 \mathrm{eV}$, which is much higher than that of the point defects present in the sample interface. It is also shown that there is a reversible change of defect density from LRS to HRS during switching, as supported by the change in $C$ from LRS to HRS. This demonstrates that the switching should not merely be an electronic process, i.e., carrier 
trapping/detrapping by fixed defects. We also identified that a change in the density of shallow defects can induce a reversible $R$ hysteresis, i.e., a switching, under slow quasistatic voltage sweeps. However, the exact nature of these defects are not yet known, and their identification continues to be a point of study.
${ }^{1}$ S. Q. Liu, N. J. Wu, and A. Ignatiev, Appl. Phys. Lett. 76, 2749 (2000).

${ }^{2}$ A. Odagawa, H. Sato, I. H. Inoue, H. Akoh, M. Kawasaki, Y. Tokura, T. Kanno, and H. Adachi, Phys. Rev. B 70, 224403 (2004).

${ }^{3}$ R. Dong, D. S. Lee, W. F. Xiang, S. J. Oh, D. J. Seong, S. H. Heo, H. J. Choi, M. J. Kwon, S. N. Seo, M. B. Pyun, M. Hasan, and H. Hwang, Appl. Phys. Lett. 90, 042107 (2007).

${ }^{4}$ M. Quintero, P. Levy, A. G. Leyva, and M. J. Rozenberg, Phys. Rev. Lett. 98, 116601 (2007).

${ }^{5}$ Y. B. Nian, J. Strozier, N. J. Wu, X. Chen, and A. Ignatiev, Phys. Rev. Lett. 98, 146403 (2007).

${ }^{6}$ K. Szot, W. Speier, G. Bihlmayer, and R. Waser, Nature Mater. 5, 312 (2006).

${ }^{7}$ S. Tsui, A. Baikalov, J. Cmaidalka, Y. Y. Sun, Y. Q. Wang, Y. Y. Xue, C. W. Chu, L. Chen, and A. J. Jacobson, Appl. Phys. Lett. 85, 317 (2004).

${ }^{8}$ A. Sawa, T. Fujii, M. Kawasaki, and Y. Tokura, Appl. Phys. Lett. 86, 112508 (2005).

${ }^{9}$ S. Tsui, Y. Q. Wang, Y. Y. Xue, and C. W. Chu, Appl. Phys. Lett. 89, 123502 (2006).

${ }^{10}$ R. Meyer, R. Waser, J. Helmbold, and G. Borchardt, Phys. Rev.
Lett. 90, 105901 (2003).

${ }^{11}$ D. S. Shang, Q. Wang, L. D. Chen, R. Dong, X. M. Li, and W. Q. Zhang, Phys. Rev. B 73, 245427 (2006).

${ }^{12}$ Y. Xia, W. He, L. Chen, X. Meng, and Z. Liu, Appl. Phys. Lett. 90, 022907 (2007).

${ }^{13}$ A. Baikalov, Y. Q. Wang, B. Shen, B. Lorenz, S. Tsui, Y. Y. Sun, Y. Y. Xue, and C. W. Chu, Appl. Phys. Lett. 83, 957 (2003).

${ }^{14}$ A. Rose, Phys. Rev. 97, 1538 (1955).

${ }^{15}$ For example, D. Dascalu, Int. J. Electron. 21, 183 (1966).

${ }^{16}$ J. J. Liu, C. Duan, W. N. Mei, R. W. Smith, and J. R. Hardy, J. Appl. Phys. 98, 093703 (2005).

${ }^{17}$ For a general review on dielectric relaxation, A. K. Jonscher, J. Phys. D 32, R57 (1999).

${ }^{18}$ J. C. Kimball and L. W. Adams, Phys. Rev. B 18, 5851 (1978).

${ }^{19}$ J. Sichelschmidt, M. Paraskevopoulos, M. Brando, R. Wehn, D. Ivannikov, F. Mayr, K. Pucher, J. Hemberger, A. Pimenov, H.-A. Krug von Nidda, P. Lunkenheimer, V. Yu. Ivanov, A. A. Mukhin, A. M. Balbashov, and A. Loidl, Eur. Phys. J. B 20, 7 (2001).

${ }^{20}$ S. Mercone, A. Wahl, A. Pautrat, M. Pollet, and C. Simon, Phys. Rev. B 69, 174433 (2004). 